\title{
Universidad e innovación en función del desarrollo local
}

\author{
Delgado Tornés, Alisa N. \\ Universidad de Granma, Cuba \\ adelgadot@udg.co.cu \\ León Téllez, C. Ibrahin Amhed \\ Universidad de Granma, Cuba \\ ileont@udg.co.cu
}

Rosales Rivera, José Q.

Universidad de Granma, Cuba

jrosalesr@udg.co.cu

\section{Resumen}

El proceso de formación del profesional universitario que se desarrolla en la educación superior como un espacio de construcción de significados y sentidos entre los sujetos participantes, implica el desarrollo humano progresivo, lo que debe ser un proceso que se reconozca como innovador, consciente y complejo, pero siempre en función del desarrollo local.

Reconocemos que las universidades deben generar innovaciones si quieren avanzar y no solo adaptarse a los cambios sociales. En el contexto cubano se plantea como imperativo en relación con el quehacer universitario, su papel en aquellos aspectos relacionados con conceptos tales como desarrollo y las representaciones sobre ciencia, tecnología e innovación, y su conexión con el desarrollo local.

En el presente artículo se exponen los principales fundamentos teóricos metodológicos y se exponen variadas experiencias sistematizadas en la red universitaria de gestión del conocimiento y la innovación para el desarrollo local en la Provincia Granma de la República de Cuba.
Palabras clave: universidad; investigación; innovación; desarrollo local;

\section{Abstract}

The process of training the university professional that develops in higher education as a space for the construction of meanings and meanings among the participating subjects, implies progressive human development, which must be a process that is recognized as innovative, conscious and complex but always depending on local development.

We recognize that universities must generate innovations if they want to advance and not just adapt to social changes. In the Cuban context, its role in those aspects related to concepts such as development and representations about science, technology and innovation, and its connection with local development is considered imperative in relation to university work.

This article presents the main theoretical and methodological foundations and exposes various systematized experiences in the university network of knowledge 
management and innovation for local development in Granma Province of the Republic of Cuba

Keywords: university; research; innovation; local development;

\section{INTRODUCCIÓN}

Uno de los retos que enfrentan las universidades en los últimos años representa la formación de profesionales con calidad mediante la perspectiva del aprendizaje permanente y la cooperación entre las instituciones universitarias y que las entidades de producción mantengan una comunicación profesional, que permita generar investigaciones cuyos resultados impacten el desarrollo local.

Los cambios culturales, educativos y sociales generados en los diferentes contextos donde el desarrollo social de la tecnociencia ha conducido en aplicar sobre la práctica, conceptos de eficiencia, calidad y exigencia en los procesos formativos universitarios que resulten, cada vez más comprometidos y en interacción permanente con la sociedad.
No se ignora que las universidades enfrentan en la actualidad, el reto de ampliar sus capacidades y dar respuesta a las exigencias sociales, a las crecientes demandas de que los profesionales en formación sean capaces de insertarse plenamente en los procesos sociales, productivos y científicos en un contexto complejo, caracterizado por las desigualdades sociales, situaciones económicas, los vertiginosos cambios tecnológicos y la diversidad sociocultural.

Dos aspectos resultan esenciales de los procesos innovativos que deben acontecer en la misión universitaria, son ellos:

- Productos o procesos sociales donde los factores epistémicos desempeñan roles primordiales en la génesis y consolidación del conocimiento

- Críticas a la tecnocracia

De forma conjunta, tres direcciones definen a los procesos innovativos y constituyen uno de los retos en la actualidad para las universidades. 
Figura 1. Direcciones de los procesos innovativos en la universidad.

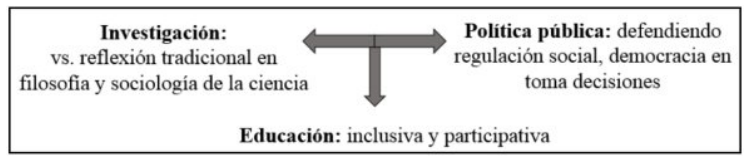

Fuente: Delgado Tornés, 2018

Sin embargo, aún algunas universidades muestran fisuras que no conducen a un desarrollo ascendente del profesional universitario debido a, la insuficiente visión dialéctica y en sistema que contribuya a tales fines; pues no siempre el personal más calificado y de experiencia que ostentan categorías superiores y grados científicos se le aprovecha de forma conveniente para la preparación de otros profesionales. Conjuntamente, en ocasiones se carece de una estrategia que debe proyectarse de modo, que entender las controversias generadas entre la ciencia, tecnociencia y la sociedad resultan esenciales.

\section{DESARROLLO}

En la actualidad, es reconocida que la producción sobre las transformaciones a nivel mundial posee impactos significativos en la concepción del hombre, además, de su relación con el entorno donde se evidencian importantes cambios en la educación superior tanto en su concepción general como a nivel de enfoques pedagógicos y contenidos curriculares. En tal sentido, la significación sobre lo axiológico y actitudinal con énfasis en la valoración cognitiva requiere de una visión más dialéctica y holística del proceso formativo de los profesionales.

Un aspecto en la calidad de la formación universitaria quizás se centre sobre el resultado de problemas crecientes que hoy enfrentamos como la crisis ecológica (Huutoniemi \& Tapio, 2014) donde desafiar los paradigmas dominantes constituye un nuevo reto (Lotz et al., 2015). Los enfoques contemporáneos de la educación superior que aún prevalecen, no tributan a la formación de un profesional capacitado para entender y responder la abrumadora acumulación de problemas ecológicos, sociales y económicos como el cambio climático, la rápida pérdida de biodiversidad, la satisfacción de necesidades sociales y escasez de agua (Rockstrom et al., 2009; Jones, 2013) por lo que quizás se carezca y sea una contradicción, capacidad de resolución para enfrentar los grandes de- 
safíos presentados con la acumulación de conocimientos producidos (Lotz et al., 2015) y los retos que tenemos en cuanto a desarrollo local se refiere.

Los cambios de paradigmas de la educación superior que responden a las condicionantes planteadas anteriormente, implican a su vez cambios en el significado de los conceptos y categorías, así como de la forma de relacionarlos. Lo que se expresa en cambios epistemológicos, axiológicos y actitudinales frente a los procesos, fenómenos o situaciones que se interpretan e investigan desde los nuevos paradigmas deben modificar la forma de desarrollar o interpretar los procesos. En este sentido, hay que reconocer que uno de los problemas más debatidos en el ámbito de los paradigmas de la educación, es la cuestión de la calidad de las acciones humanas para alcanzar mejores y sostenibles resultados donde se han acuñado diferentes categorías que intentan expresar de la mejor manera dicha intencionalidad, provocando la asunción de posiciones diversas en cuanto al diseño del proceso formativo de profesionales universitarios.
El proceso de formación del profesional universitario que se desarrolla en la educación superior como un espacio de construcción de significados y sentidos entre los sujetos participantes, implica el desarrollo humano progresivo, lo que debe ser un proceso que se reconozca como innovador, consciente y complejo, pero siempre en función del desarrollo local.

La formación profesional debe constituir, por lo tanto, el proceso en el que los sujetos desarrollan el compromiso social y profesional, flexibilidad ante la cultura, trascendencia en su contexto, ante todo local, toda vez que elevan su capacidad para la reflexión divergente y creativa, evaluación crítica y autocrítica, solución de problemas, toma decisiones y adaptarse flexiblemente al entorno cada vez más cambiante. Se asume que, alcanzar una integralidad en la formación profesional a nivel universitario implica ante todo formar un profesional comprometido con su profesión y la sociedad en que se inserta, adoptando postura flexible y trascendental, independientemente de la especificidad que impone sus variados contextos de 
actuación. Justamente en este camino resulta imprescindible fomentar redes que coadyuven a tales propósitos.

\section{RED GESTIÓN UNIVERSITARIA}

DEL CONOCIMIENTO Y LA

INNOVACIÓN PARA EL

DESARROLLO LOCAL EN LA

\section{UNIVERSIDAD DE GRANMA}

EI CEDDEL ${ }^{1}$, es una de las instituciones que investiga sobre DesarroIlo Local en Cuba, asume como perspectiva metodológica fundamental la cualitativa, respondiendo de esta forma a las necesidades investigativas que permiten el estudio de las relaciones existentes entre los diferentes actores sociales, las instituciones y los gobiernos locales; así como el nexo que se establece entre estos actores y las tecnologías sociales disponibles.

Reconocemos que las universidades deben generar innovaciones si quieren avanzar y no solo adaptarse a los cambios sociales. En tal sentido en su trabajo sobre la cultura innovadora de las universidades
Marina Tomás (2006) ubica tres claves para los procesos de atención específica al cambio y la innovación; en la primera clave le sitúa a la universidad el reto de que no solo genere conocimiento, sino que construya en su interior una cultura innovadora de la que se apropie todo aquel que pase por ella y transmita este conocimiento y cultura a la sociedad. Las otras dos claves se relacionan con la producción de conocimientos y los programas de formación permanente.

En la actualidad a escala municipal en la Provincia de Granma de la República de Cuba, existe un conjunto de barreras que obstaculizan la gestión e integración en función del desarrollo local.

Esto requiere que el papel de las universidades en el desarrollo local y territorial, introduzca en la práctica social los cambios conceptuales acerca del desarrollo y las representaciones sobre ciencia, tecnología e innovación, y su conexión con el desarrollo local.

1. Centro de Estudios de Dirección y Desarrollo Local, de la Universidad de Granma 
En el contexto cubano se plantea como imperativo en relación con el quehacer universitario la necesidad de vincular su potencial científico, a través de sus procesos sustantivos en un accionar más proactivo hacia la gestión del conocimiento y la innovación en función del desarrollo local, lo cual ha sido expresado en sus objetivos estratégicos de lograr impactos de la educación superior en el desarrollo local, en base a lo establecido en la Política de Desarrollo Económico y Social Territorial. Incentivando el desarrollo de más proyectos de $\mathrm{i}+\mathrm{D}+\mathrm{l}$ en el ámbito del desarrollo socio comunitarios, articulados con programas y proyectos de desarrollo económico gestionados por las universidades, centros de investigación y CUM, que deberán aportar tecnologías a los municipios y las capacidades de absorción correspondientes, con impacto reconocido.

Esto implica incluir dentro de los procesos sustantivos de las universidades la línea estratégica que enfoca al papel que deben jugar como agentes o actores claves en los procesos de gestión del conocimiento y la innovación a nivel local.
Ante este imperativo surge el problema relacionado de ¿cómo puede la universidad con sus potencialidades en el ámbito del conocimiento contribuir al desarrollo local?

Para la Universidad de Granma el desarrollo local constituye un proceso complejo de construcción colectiva y participativa, que implica asumir un enfoque complejo y holístico que debe articular las propuestas teóricas y otras innovaciones, resultantes de sus proyectos, con los conocimientos, procesos y experiencias que acontecen a escala local. (Arias Guevara y Labrada, 2008)

El principal desafío que enfrentan los municipios para elaborar un programa efectivo de desarrollo, es el de saber cómo diseñar y aplicar sistemas de gestión capaces de fomentar y conciliar los tres grandes objetivos que, en teoría, llevarían al desarrollo sustentable: el crecimiento económico, la equidad (social, cultural, económica y ambiental) y la sustentabilidad. 
La institucionalización de los procesos universitarios en los espacios locales de nivel municipal en la Provincia de Granma, surgió como consecuencia de la emergencia en estos contextos de las Sedes Universitarias Municipales (SUM) y sus desarrollos condujeron posteriormente a los actuales Centros Universitarios Municipales (CUM). Desde el año 2005 este ha sido un proceso de incorporación paulatina de las funciones sustantivas de las instituciones de educación superior, que ha venido ofreciendo oportunidades excepcionales para desarrollar las capacidades locales y la gestión del conocimiento y la innovación, orientada al mejoramiento de las condiciones tecnológicas, sociales y culturales de estos espacios (Morales M, 2012).

Desde este punto de vista la Universidad de Granma se convierte en un actor clave, en esta provincia, que a partir de sus funciones sustantivas, diseñe herramientas capaces de propiciar efectividad en la gestión del desarrollo en el contexto donde están enmarcadas, basado en un enfoque de trabajo en red universitaria de gestión del conocimiento para el desarrollo local.
La Universidad de Granma posee un rico precedente que desde lo académico se ha venido desarrollando en nuestro país, entre los que se puede destacar la Red Gestión Universitaria Conocimiento y la Innovación del Desarrollo, proyectos de investigación de los centros de estudio e investigación asociados a las diferentes universidades en el país; así como publicaciones en revistas académicas, como la Universidad de la Habana y de la revista GUCID. Es pertinente reconocer, en este sentido, los aportes de los Centros Universitarios Municipales (CUM) y otros del Centro de Estudio de Dirección y Desarrollo Local de la Universidad de Granma.

En este espacio procuramos abordar algunas de las experiencias de la Universidad de Granma derivadas de gestión de la Red Gestión Universitaria Conocimiento y la Innovación del Desarrollo enfocadas al desarrollo local.

En su estructuración se incorporaron diferentes enfoques y teorías desarrolladas basada en los estudios sobre constitución de redes. A 
partir de un enfoque multidisciplinario, reconociendo la complejidad y el significado de la relación multiactores y multinivel a partir del espiral en que se dan esas relaciones. El trabajo de la red se constituye en una acción estratégica para que el modelo de gestión asumido constituya guía de adaptación a las distintas realidades locales.

En su concepción teórica se asumen posturas que reafirman su necesidad (Coraggio J, 2002), sobre los vínculos Universidad - Desarrollo Local, asì como los de (Casa y Luna, 2011) que explicitan las vías de como se pueden articular estas redes a partir de cooperación de relaciones bilaterales. En el contexto granmense lo relacionado al desarrollo local desde la gestión del conocimiento constituye una prioridad para la Universidad de Granma.

Orozco (2005) señala que, la gestión del conocimiento promueve la generación, colaboración y utilización del conocimiento para el aprendizaje organizacional, generando así nuevo valor y elevando el nivel de competitividad con miras a alcanzar sus objetivos con eficiencia y eficacia; así pues se generali- zan las mejores prácticas, a la vez que facilita la generación de nuevos conocimientos y su materialización en productos y servicios. La gestión del conocimiento es un proceso relacionado de una u otra forma con la captación, la estructuración y la transmisión del conocimiento y que la importancia que se le atribuye actualmente está condicionada por variables sociales, como la economía, la cultura y la política.

Por su parte, la innovación social designa nuevas formas organizacionales y prácticas sociales que contribuyen al mejoramiento de una situación o dan una respuesta inédita a una necesidad colectiva. En la misma está presente la alianza entre diversos actores a nivel local, existiendo, de este modo, un vínculo entre innovación social y desarrollo local. Esto suscita un interés creciente ya que un aspecto importante de la innovación social reside en la generación de relaciones de cooperación entre diversos actores.

El objetivo general de la Innovación Social y el Desarrollo Local está encaminado en promover el compromiso para participar activa- 
mente en el desarrollo del proceso, así como favorecer cambio de mentalidades y la generación de espacios de enseñanza - aprendizaje que permitan una reflexión profunda y delimitar acciones concretas que favorezcan el desarrollo local; además de contribuir a la generación de oportunidades productivas en las cuales se incorpore el potencial de cada región.

Las innovaciones sociales tienen dimensiones tangibles (creación de puestos de trabajo, nuevos productos, mejoramiento de las condiciones de vida, formas organizacionales, etc.), pero poseen también dimensiones intangibles: confianza, vínculos, información, desarrollo de las capacidades propias (De la Maza, 2003).

Estos elementos conducen a señalar que por la naturaleza que encierra la gestión del conocimiento y la innovación social, propician que esta articulación posibilite las bases estructurales y el alcance de esta red universitaria.
Han sido estos tres elementos como plataforma teórica del diseño y dinámica que ha movido a la red de la Universidad de Granma hasta convertirse en un elemento dinamizador de la gestión del desarrollo local. En su composición se incorporan los fundamentos del trabajo en redes a partir de los presupuestos teóricos sustentados en la gestión del conocimiento y la innovación social. Dentro de la Comunidad Universitaria forman parte activa de la red; la Dirección de Gestión del Conocimiento -adscripta a la Vicerrectoría de Investigación y Postgrado-, los Centros de Estudio de Dirección y del DesarroIlo Local, los Centros Universitarios Municipales (CUMs) con sus respectivos departamentos de Desarrollo Local y el entorno gubernamental del nivel provincial y municipal. Por lo que su funcionamiento se basa en un enfoque de multiactores y multinivel.

La dirección para cumplir su encargo y objetivos se sustenta en un mapa estratégico que sintetiza las acciones a desarrollar por parte de la red, para cumplir con el encargo social visto desde el vínculo univer- 
sidad entorno socio- productivo gubernamental. En él se detallan las principales direcciones del trabajo de la red y se orientan las principales acciones que en correspondencia con ello se deben emprender considerándose dentro de ellas: los procesos de investigación científica que han permitido el perfeccionamiento del trabajo de los grupos científicos y líneas de investigación con prioridad en la gestión de proyectos.

Otra dirección está relacionada con la superación posgraduada, con vistas a que la misma a través de su concepción potencie el proceso de desarrollo local. En este sentido se ha diseñado programas de maestría, pudiendo destacar el programa de Maestría en Desarrollo Socioeconómico Local y el programa de Maestría en Contabilidad Gerencial. El primero transita por su segunda edición y el segundo por la quinta edición; así como dos programas de diplomados, uno en Gestión del Desarrollo Local y otro relacionado con la Gestión Sociocultural del Desarrollo Local.
Una tercera dirección se relaciona con las acciones a desarrollar en relación con la consolidación de las acciones de asesoría a los gobiernos municipales en lo relacionado con los programas de desarrollo integral, los proyectos de desarrollo local y la gestión cooperativa.

Como parte de su accionar, en los últimos cinco años la red ha desarrollado diferentes acciones que han sido el resultado de un proceso de planificación, que tuvo como punto de partida, el cumplimiento de los objetivos de la universidad relacionados con el desarrollo local. En la actualidad se han venido realizando evaluaciones, talleres y otros espacios de diálogo que han contribuido a la sistematización de las acciones realizadas por la red y visibilizar no solo los resultados en estas direcciones, sino a identificar lo que aún queda pendiente por realizar.

Indudablemente que la memoria resultado del proceso contribuye a la visibilidad de resultados y a la posibilidad de su introducción como práctica en los diferentes contextos. 
Sin duda estas acciones han contribuido al logro de impactos positivos en el orden organizacional y del perfeccionamiento de la conducción de los gobiernos locales, así como en el perfeccionamiento de políticas relacionadas con el desarrollo local en el contexto de los municipios granmenses de Bayamo, ManzaniIlo, Guisa, Buey Arriba, Pilón, Río Cauto, Jiguaní, Cauto Cristo.

Justamente la red universitaria ha contribuido a que las iniciativas de desarrollo local revitalicen el vínculo entre las autoridades centrales y la administración provincial y municipal, brindando mayor protagonismo a los actores locales en la búsqueda de soluciones a sus propios problemas.

En función de lo anterior la red universitaria ha ido procurando progresivamente una interrelación dinámica entre la universidad y los gobiernos en pro del desarrollo local, asignándole a la universidad el rol como factor mediador de la constitución e integración de todos los actores locales.
De este modo recuperamos lo planteado por Casas R, et al (2003) de que las redes se construyen mediante intercambios entre un conjunto de actores que en relación a la gestión del conocimiento comparten el mismo en función de contribuir al desarrollo local. Lo cual reafirma el papel que deben jugar las universidades en la generación y distribución del conocimiento.

En este sentido la Red Gestión Universitaria Conocimiento y la Innovación del Desarrollo Local de la Universidad de Granma, tiene como misión desarrollar actividades que permitan la difusión del conocimiento desde la ciencia y la innovación, relacionando a la universidad y los CUMs con su entorno para responder a las necesidades de las instituciones públicas, productivas y de servicios con el fin de contribuir a la solución de problemas locales.

La universidad tiene que estar articulada a las necesidades del desarrollo local y debe distinguirse por ser una institución generadora de conocimiento presto a introducir en la práctica social. Pero además 
contribuir al proceso del desarrollo local, en el que los sujetos desarroIlen el compromiso social y profesional, flexibilidad ante la cultura, trascendencia en su contexto, toda vez que eleven su capacidad para la reflexión divergente y creativa, evaluación crítica y autocrítica, solución de problemas, toma decisiones $y$ adaptarse flexiblemente al entorno cambiante.

La comprensión del desarrollo, entendido en esta escala de lo local, coloca como fundamental la coordinación de acciones de los gobiernos municipales con los centros universitarios y las universidades centrales, en busca del mejoramiento de los mecanismos de gestión institucional, propiciadores de una adecuada orientación del conocimiento y la innovación, que destaque la contribución de la universidad a la solución de los problemas de sus comunidades. (Morales M, 2012)

En la Universidad de Granma, como parte de su estrategia para la ciencia y la innovación, desarrolla tres líneas priorizadas a nivel central, una de ellas la constituye la gestión de los procesos relaciona- dos con el desarrollo local, con vista a incidir desde la gestión del conocimiento al fortalecimiento de este proceso.

La Red Gestión Universitaria Conocimiento y la Innovación del Desarrollo Local en la Universidad de Granma es expresión de una universidad científica, tecnológica, humanista y comprometida con el desarrollo social de la provincia, facilita a nivel local el desarrollo de sus procesos sustantivos y desarroIla sus investigaciones, procesos de capacitación y extensión universitaria que prioricen los gobiernos locales y a los actores sociales para conducir y protagonizar las transformaciones que necesita el desarrollo local.

Entre los resultados más significativos de la Red se encuentran los siguientes:

- Implementación de un sistema de gestión integral en empresas del Ministerio de la Industria Alimenticia (MINAL).

- Índice sintético de desarrollo municipal y de la Provincia Granma.

- Sistema de monitoreo y evaluación del subsistema económico del municipio Río Cauto. 
- Índice sintético de desarrollo local para la comparación intermunicipal.

- Perfeccionamiento de la gestión financiera en empresas del Ministerio de la Industria Alimenticia (MINAL) en la provincia.

- Diseño e implementación del sistema de gestión asociado al Plan de Desarrollo Integral (PDI). Perfeccionamiento de la gestión del desarrollo local a escala municipal y provincial. (Metodologías y procedimientos).

- Identificación de los factores que inciden en la comercialización de productos agrícolas en la provincia Granma.

- Diseño del observatorio científico tecnológico de desarrollo local de la Universidad de Granma.

- Implementación de un sistema integral de dirección estratégica empresarial con orientación al desarrollo local.

- Sistema de información territorial y metodología por dimensiones con enfoque intra e interregional.

- Modelo para la selección y jerarquización de proyectos de Iniciativa Municipal Desarrollo Local (IMDL)

- Análisis de la dinámica y estructura de la economía de la provincia Granma

- Acciones para desarrollar capacidades de emprendimiento en el sector productivo, cooperativas, campesinos.

En el contexto municipal con la participación de los Centros Universitarios Municipales (CUMs), se ha desarrollado un acompañamiento al sector productivo en sus iniciativas de emprendimiento que conducen a encadenamientos productivos, procesos que generan nuevos productos y servicios, lo cual se traduce en una mayor satisfacción de necesidades, tanto para el consumo humano como animal.

De otra parte, encontramos el fomento de minindustrias para la producción de piensos, con desechos y otros residuales de cosechas, que contribuyen a la sostenibilidad de la cadena de la producción de huevo en el municipio Bayamo.

En los municipios Bayamo, Guisa y Jiguaní se participa de forma activa en el Programa de Innovación Agraria Local, cuya implementación se ha extendido a 169 fincas y se han capacitado a 2340 actores para 
la innovación rural. Se ha logrado el establecimiento de 2 minindustrias de procesamiento de conservas en municipios de Jiguaní.

El proyecto AGROCADENAS realiza acciones en los municipios Jiguaní, Cauto Cristo y Bayamo contribuyendo a la gestión de 21 proyectos en entidades relacionadas con las cadenas de carne vacuna y del maíz.

El Programa Mundial de Alimento, se inserta en la cadena del frijol en los municipios Campechuela y Pilón, potenciando procesos innovativos en 3 cooperativas de cada municipio.

El proceso de articulación de la Red con los gobiernos y actores locales ha contribuido al despliegue del perfeccionamiento de las relaciones de coordinación de las tareas. Para ello existe un sistema de trabajo, que se ha ido consolidando progresivamente para articular el trabajo del gobierno, Centros Universitarios Municipales (CUMs) y otros actores productivos, sociales y políticos de los municipios. El mismo está organizado a partir de las relaciones que se establecen, en un grupo de trabajo liderado por el gobierno, donde se planifican las acciones a desarrollar y por ese sistema de trabajo se fiscaliza, monitorea y controlan las acciones con el objetivo de encaminar las mismas al logro de una participación integrada de todos los actores que intervienen en la transformación del territorio y mantener la sostenibilidad del desarrollo local.

Ejemplos visibles aparecen en los diferentes municipios. En Bayamo, Campechuela, Jiguaní, Rio Cauto, Yara y Buey Arriba.

El Centro de Estudio de Dirección y Desarrollo Local (CEDDEL), de la Universidad de Granma, con vistas a obtener mayor impacto de sus resultados investigativos, trabaja de forma articulada con el gobierno provincial y los gobiernos municipales, liderando el trabajo metodológico del Grupo Provincial de Desarrollo Local y en particular para el municipio Bayamo. A través de este acompañamiento, se contribuye a la superación y capacitación 
de los gobiernos locales y se ha logrado, la introducción de varios resultados de investigación en la práctica social que constituyen tecnologías de gestión del desarrollo local.

Con igual propósito se diseñaron asesorías en gestión de proyectos, metodología para los diagnósticos municipales para el Plan de Desarrollo Integral, estudios de factibilidad, entre otras acciones.

Se han conformado dos espacios con carácter viable y sostenible dirigido a la visualización, sistematización y socialización de los resultados. Nos referimos al Congreso Cubano de Desarrollo Local, que se distingue por su prestigio académico en el tema, por la participación de los gobiernos locales y diversos actores locales, por haber realizado varias ediciones que enaltecen su reputación científica; los resultados de estos congresos han sido publicados en la Revista de Desarrollo Local de la UDG, que ya cuenta con una tradición de varios números, y que indudablemente han contribuido no solo a dialogar las problemáticas del desarrollo local, sino a socializar experien- cias y aprendizajes útiles para el desarrollo local.

\section{CONCLUSIONES}

Resulta necesario que la estrategia concebida por las universidades diseñe los niveles de desarrollo profesional para cumplir el encargo de constituirse como un profesional en constante proceso de autodesarroIlo científico, cívico y cultural entre otras razones al servicio de su contexto local.

El entorno económico, político y sociocultural local condiciona los procesos educativos en las universidades; sin embargo, la universidad no debe asumir una posición adaptativa al entorno, sino que debe investigarlo con profundidad para generar los procesos de su transformación.

El perfeccionamiento del proceso de formación profesional universitaria debe tener en cuenta los avances científicos y tecnológicos, redefinir o rescatar los valores humanos y sociales, centrar los procesos en la formación integral de las personas, hacer de los centros educativos verdaderos proyectos 
culturales, formar líderes para producir la transformación, partir de la realidad que ofrece el entorno y definir los cambios a la misma, investigar sobre los entornos socioculturales y definir alternativas de solución a los problemas encontrados, construir nuevos modelos formativos y operarlos en los centros universitarios mediante estrategias científicas que transformen las formas tradicionales de administración para generar una cultura organizacional a favor del desarrollo local.

El empeño de que esos procesos formativos respondan de manera más pertinente a las exigencias sociales, económicas y productivas del desarrollo local nuestros países, impone la necesidad de que los sujetos en formación demuestren con mayor efectividad el resultado de sus aprendizajes en su actuación profesional a través de la innovación.

La sistematización de las experiencias de la red evidencia transformaciones en la gestión del conocimiento en función del desarrollo local en municipios y provincia de Granma.
El enfoque basado en los fundamentos de la gestión del conocimiento y la innovación social posibilitan el articulado multiactores y multinivel en una red ha permitido la presentación de experiencias exitosas vinculadas a la gestión del desarrollo local en el contexto del territorio granmense.

La red demuestra que las universidades pueden contribuir a incentivar la proyección local del conocimiento ampliando su capacidad de fomentar el bienestar humano en los territorios donde se encuentran enclavadas al propiciar una herramienta que posibilita gestionar el conocimiento en función del desarrollo de los territorios.

La red con un enfoque basada en los fundamentos de la Gestión del Conocimiento y la Innovación Social posibilitan el articulado multiactores y multinivel permitiendo la interacción en función de aplicar experiencias exitosas vinculadas a la gestión del desarrollo local en el contexto del territorio.

La sistematización de las experiencias de la red evidencia trans- 
formaciones en la gestión del conocimiento en función del desarrollo local en municipios de la provincia Granma. Estas se hacen visibles a partir del mejoramiento de los indicadores sociales reflejados en la aplicación de herramientas de gestión para el desarrollo local.

\section{BIBLIOGRAFÍA}

Alburquerque, Manuel. 2004. Desarrollo económico local y descentralización en América Latina. Investigación de la CEPAL. Revista de a CEPAL Nº 82. Abril.

Albornoz, M. (2010). Ciencia, Tecnología y Universidad en Iberoamérica. Buenos Aires: Eudeba.

Arias Guevara, María y Ciro Labrada Silva (Comp.) 2008. Estudios sobre desarrollo local e innovación social. Editorial Academia. La Habana.

Boffill Vega Sinaí. (2013) LA gestión del conocimiento y su contribución al desarrollo local en Cuba. Folletos Gerenciales del MES

Boisier, Sergio (2005). Un ensayo epistemológico y axiológico sobre gestión del desarrollo local: conocimientos y valores. Santiago de Chile.

(2005). ¿Hay espacio pa-

ra el desarrollo local en la globalización?. Revista CEPAL, No 86, Agosto, p. 47-62.

Cantú Delgado, H. (2009). Desarrollo de una cultura de calidad. México: Mc Graww Hill.
Casa R. y Luna M. (2011) De redes y espacios del conocimiento. En. Nuñez Jover Jorge. Universidad y Desarrollo Local: contribuciones latinoamericanas

CEPAL (2016), "Desarrollo social inclusivo. Una nueva generación de políticas para superar la pobreza y reducir la desigualdad en América Latina y el Caribe", LC.L/4056/Rev.1. ONU, enero.

Coraggio, José Luís. 2003. El papel de la teoría en el desarrollo local. Universidad Andina, Quito.

Dájer, Jesús (2006) Modelo para la gestión del conocimiento en los Destacamentos Fronterizos del país. Tesis presentada en opción al grado de Doctor en Ciencias Técnicas, UMCC, septiembre, 2006. 104p.

De la Maza, G. (2003). "Innovaciones ciudadanas y políticas públicas locales en Chile". Reforma y Democracia, 26, junio (www.clad.org.ve).

Delgado Tornés, Alisa .N. (2018). Ciencia, tecnología y sociedad. El cambio conceptual básico en la interpretación de la ciencia y la tecnología. Conferencia impartida en la Escuela de Posgrado, Universidad Nacional "San Luis Gonzaga de Ica", Ica-Perú. Julio, 2018.

Figueroa Alonso Galia. (2013) Las Ciencia Sociales y la articulación del modelo económico y social cubano. Revista Universidad de La Habana, julio- diciembre 2013.

Francés Wesley (2009) Social Innovation Generator (SIG), University of Waterloo 2009. 
Garea, B y Curbelo, A. (2008) Capacidad Innovadora: De lo empresarial a lo territorial - de lo territorial a la empresa. Centro de Gerencia de Proyectos y Programas Priorizados, La Habana, p.9

González, R. (2009). La gestión del desarrollo local con un enfoque integrador. Términos para un debate. Revista Internacional La Nueva Gestión Organizacional, 4(8), 44-72.

Huutoniemi, K. \& Tapio, P. (2014). Transdisciplinary sustainability studies: A heuristic approach. New York: Routledge.

Jones, D.R. (2013). "The Biophilic University": A de-familiarizing organizational metaphor for ecological sustainability? Journal of Cleaner Production: 48, 148155. DOI: 10.1016/j.jclepro.2013.02.019

Lotz, S.H., Walsisitka, A.E.J., Kronlid, D. \& McGarry, D. (2015). Transformative, transgressive social learning: rethinking higher education pedagogy in times of systemic global dysfunction. Current Opinion in Environmental Sustainability; 16:73-80. DOI: 10.1016/j.cosust.2015.07.018

Martínez, T, Alicia; Expósito G. E, Delgado, T. Alisa. (2015) La red desarrollo local como estructura de relacionamiento social y funcionamiento de la ciencia, la innovación y las estructuras de los gobiernos locales. El caso de la Universidad de Oriente. En Libro Universidad, conocimiento, innovación y desarrollo local. Compilador Jorge Núñez Jover. Editorial Universitaria Félix Varela, La Habana, 2014.

MES (2018) Objetivos estratégicos del 2017. Documento del Ministerio de Educación Superior, La Habana.
Morales M, (2008). Desarrollo local, desafíos al conocimiento y la innovación. Taller de Formación de Investigadores, marzo, Cojimar, La Habana.

(2012) Retos de la Gestión Universitaria del conocimiento y la Innovación para el Desarrollo Local. Revista Congreso Universidad. Vol. I, No. 1

Núñez, J., Montalvo, F. y Pérez, Ones (2007): "Universidad y desarrollo social basado en el conocimiento: nuevas estrategias desde lo local" recuperado de: http://developinguniversities.blogsome.com.

Núñez Jover Jorge. (2013) La ciencia universitaria en el contexto de los cambios en el modelo económico social: lecciones del pasado y miradas hacia adelante. Revista Universidad de La Habana, julio- diciembre.

(2014). Universidad, conocimiento, innovación y desarrollo local. La Habana, Editorial Universitaria Félix Varela. pp.292.

Orozco E. (2005), Conferencias sobre Gestión del Conocimiento. Consultoría Biomundi, Ministerio de Ciencia y Tecnología, La Habana.

Rockstrom, J. \& et al. (2009). A safe operating space for humanity. Nature; 461, 472-475. DOI: 10.1038/461472a

Rosales Rivero, J. (2017), El impacto en los resultados científicos y tecnológicos. Aproximación para su evaluación. Conferencia impartida en Universidad de Granma.

Tomás, M. (coord.) (2006). Reconstruir la universidad a través del cambio cultural, Bellaterra, Servei de Publicacions de la UAB. 
UNESCO. La educación superior en el siglo XXI: Visión y acción. En: CONFERENCIA MUNDIAL SOBRE LA EDUCACIÓN SUPERIOR. Disponible en: www.taringa.net/posts/info/8148984, 1998. Acceso en: 15 de marzo 2014.

Vallaeys, François. ¿Qué es la Responsabilidad Social Universitaria? Disponible en: www.cedus.cl/files/RSUusb.pdf, 2012. Acceso en 15 de marzo 2014, p. 3

Universidad de Granma (2017) Informe GUCID. Documento del CEDDEL.

Universidad de Granma (2018) Informe de auto evaluación del CEDDEL para la evaluación institucional. Documento del CEDDEL. 